\title{
EFFECIENCY OF DIFFERENT INJECTION OBTURATION TECHNIQUES IN PULPECTOMIZED DECIDUOUS TEETH USING RADIOGRAPH
}

\author{
Dilan Shahgan Mohamed Taher ${ }^{*}$ and Abdulkareem R. Al-Mezouri ${ }^{* *}$ \\ *Dept. of Pedodontic, College of dentistry, University of Duhok, Kurdistan Region- Iraq. \\ ** Dept. of Conservative, College of dentistry, University of Duhok, Kurdistan Region- Iraq.
}

(Received: September 15, 2019; Accepted for Publication: November 20, 2019)

\begin{abstract}
Objective: This study aimed to evaluate and compare the quality of different obturation techniques in primary teeth (navitip, endodontic pressure syringe, insulin syringe and reamer as a control) by using peri-apical radiograph.

Materials and Methods: Eighty extracted human lower primary second molar teeth with minimum $7 \mathrm{~mm}$ root length were randomly divided in to four groups (Group one-navitip, Group two-endodontic pressure syringe, Group three - insulin syringe, Group four- reamer) were included in the study. Peri apical radiograph images were taken after the obturation for each group.To evalute over filling, under filling and optimal filling.

Result: The results showed no significant differences between the four groups for the length of obturation $(P>0.05)$. Navitip and endodontic pressure syringe showed the best results for the length of obturation (50.0\% optimal fillings) and insulin syringe showed (45.0\% optimal fillings) for the length of obturation. Reamer showed $(\mathbf{4 0 . 0 \% )}$.

Conclusion: The studies showed that all obturation techniques used didn't provide ideal obturation with nearly comparable obturation quality for the techniques.
\end{abstract}

KEY WORDS: Primary teeth, pulpectomy, root canal obturation, zinc oxide eugenol

\section{INTRODUCTION}

D ental caries is one of the most common diseases of the primary dentition. Maintaining the integrity of primary teeth until its normal exfoliation is the primary goal of pediatric dentistry (Dandashi et al., 1993).

Preservation of primary dentition is important for orofacial development as it helps to maintain the space for eruption of permanent teeth, aids in masticatory functions, phonation, and swallowing (Markovic et al., 2005).

In 1879, Witzel's pulpotomy technique advocated amputation of infected tissue and retention of devitalized pulp tissue in the canals which was sealed with strong antiseptic cement. The treatment of nonvital pulps with pulpectomy was initially questioned due to fear of damage to the erupting successors and the clinical difficulty of the procedure (Gesi and Bergnholtz, 2003).

The ultimate goal of endodontic obturation has remained the same for the past 50 years: to create a fluid-tight seal along the length of the root canal system, from the coronal opening to the apical termination (Jha etal, 2011). For this purpose, several techniques have been used for the filling of material into primary tooth root canals. An ideal filling technique should assure complete filling of the canal without overfill and with minimal or no voids (Guelmannetal, 2004). It is important to select an obturation technique that offers consistency and is easy to use (Gutmann etal, 2010).

Endodontic pressure syringe, using the technique described by Greenberg (1963) and following the manufacturer's recommendation, the standardized mixture was injected into the simulated canals. This apparatus consists of a syringe barrel, threaded plugger, wrench and threaded needle. The needle is inserted into the simulated canal until wall resistance was encountered. Using a slow, withdrawing-type motion, the needle was withdrawn in 3-mm intervals with each quarter turn of the screw until the canal can be visibly filled at the orifice with 
zinc oxide eugenol paste. (Aylard and Johnson, 1987).

Insulin syringe with needle gauge 30 is used in the placement of homogeneous mixture of ZOE. The insulin syringe is loaded with zinc oxide eugenol then the needle is inserted into the canal. The material was then pressed into the canal and while doing so, the needle was retrieved from the canal outward while continuing to press the material inside (Nagar et al., 2011).

NaviTip (NaviTipTM, Ultradent), a thin flexible metal tip was introduced to deliver root canal sealers. The greatest advantage of NaviTip is that its ends are rounded, it is rigid at the shank region, which prevents further bending and the end of the tip is flexible to facilitate navigation even through curved canals. NaviTips are available in 29 and 30 gauges. They are colour coded depending on the length-17mmwhite, $21 \mathrm{~mm}$-yellow, 25mm-blue, and $27 \mathrm{~mm}$-green. This technique is fast and user-friendly (Guelmann et al., 2004).

The Incremental Filling Technique, was first used by Gould in 1972. An endodontic remear, corresponding to the size of the canal, with rubber stop was used to place a thick mix of zinc oxideeugenol paste into the canal.

The ultimate goal of endodontic obturation has remained the same for the past 50 years: to create a fluid-tight seal along the length of the root canal system, from the coronal opening to the apical termination (Jha et al, 2011). For this purpose, several techniques have been used for the filling of material into primary teeth root canals. An ideal filling technique should assure complete filling of the canal without overfill and with minimal or no voids (Guelmann et al, 2004).

\section{MATERIALS AND METHODS}

Study was conducted in the department of Pediatric and Preventive Dentistry at the College of Dentistry /University of Duhok. In this experimental invitro study eighty extracted human lower primary second molar teeth or upper primary second molar teeth with minimum $7 \mathrm{~mm}$ root length were included. Teeth with pathological root resorption (external and/or internal); external and/or internal furcation perforation; Pulpotomy and pulpectomy teeth were excluded from the study. Teeth were washed under tap water after extraction and then cleaned mechanically with ultrasonic scaler to remove hard deposit and soft tissues and then stored in $0.1 \%$ thymol solution at room temperature in 60 flat- bottom blood collection tubes with screw cap, (Berechet etal, 2018); (Elnagar etal, 2018).

Teeth were washed with distilled water and de- coronated at Cement- Enamel Junction (CEJ) by using diamond disc. The working length for all the canals were determind by size \#10 K- file (Dentsply Malliefer, Ballaigues, Switzerland) $2 \mathrm{~mm}$ short of radiographic apex from a standardized digital plain radiograph by arranging all the samples on the radiographic film, this step done to ensure standardization, the dose of radiograph given as a mass of 45 milliamper per second, and the power was $45 \mathrm{~K} . \mathrm{V}$.

The lengths of distal canals distobuccal\& distolingual were at minimum $7 \mathrm{~mm}$. Both distal canals (Type III according to Weine classification, two separated canals)

The canals were debrided and enlarged by using rotary ENDO_MATE engine TS2 (NSK) using Pro Taper file F1,F2,F3 at speed $300 \mathrm{rpm}$ and torque 2. Canals were irrigated with Sodium hypochlorite $0.5 \%$. Next, the canals were dried with absorbent paper points size F3 and were divided in to four groups each group contain 20 canals according to obturation technique.

Group A: canals were obturated using navitip size 30. Obturation time was measured using a digital chronometer at the beginning of insertion of material (Gordon et al., 2005), 1 scoops of powder and 3 drops of liquid. The metal tip was placed into the canal, a rubber stop was adjusted to the predetermined measurement and ZOE will be expressed. Once backfill of filling material occurred, the canal was assumed filled.

Group B: canals were obturated using Endodontic Pressure Syringe 30gauge needle, according to the manufacturer's instructions. Obturation time was measured using a digital chronometer at the beginning of insertion (Gordon et al., 2005). The mechanical nature of the pressure syringe (which operates by a screw mechanism) expressed the mixture of $\mathrm{ZoE}$. The powder liquid ratios was (1:3) (Reddy etal., 2015). Through a 30-gauge needle. The needle was inserted into the root canal and using a slow, withdrawing-type motion, the needle was withdrawn in $3 \mathrm{~mm}$ intervals with each quarter turn of the screw until the canal was visibly filled at the orifice.

Group C: canals were obturated using insulin syringe (needle; 30-gauge, $8 \mathrm{~mm}$ length; 
DispoVan). Zinc oxide eugenol paste mixed The powder liquid ratios was (1:3) then loaded into the insulin syringe and the needle was inserted into the canal. The material was pressed into the canal. The needle was retrieved from the canal outward while continuing to press the material inside. Finally, at the orifice, more materials were pressed using a moist cotton pellet.

Group D: canals were obturated using endodontic reamer size 30 with rubber stop. Tow scoops of zinc oxide eugenol powder and tow droops of liquid mixed till thick mixture was obtained. Obturation time was measured using a digital chronometer at the beginning of insertion of material for all the groups (Gordon et al., 2005). Length of the endodontic reamer equaled the predetermined root canal length minus $2 \mathrm{~mm}$. Reamer carried zinc oxide eugenol to the canals. Additional increments were added until the canal was filled to the cervical area. After 24 hours peri apical radiograph had been taken for all groups (Moutaz et al., 1993) (Table 1and 2) (Figure 1).
Scores quality of obturation based on the following criteria as given by (Coll and Sadrian, 1996).

- Score (1) under filling: All canals filled more than $2 \mathrm{~mm}$ short of the apex.

- Score (2) optimal filling: Canals having obturation material ending at the radiographic apex or up to $2 \mathrm{~mm}$ short of apex.

-Score (3) over filling: Any canal showing obturation material outside the root apex.

\section{RESULTS}

The result showed Navitip and Endodontic Pressure Syringe had optimum length of obturation (50.0) as acompared to insulin syringe and reamer which were (45.0) (40.0) respectively (Table 1, Figure 1).

Fishers' exact test was performed, that there is no a statistically significant difference in the score between group A and control group $(\mathrm{P}=0.218)$, group $\mathrm{B}$ and control group $(\mathrm{P}=0.340)$, group $\mathrm{C}$ and control group $(\mathrm{P}=1.000)$ as shown in Table 2.

Table (1): Overall comparison of score of length of obturation in four study groups by Fishers' exact test was performed for statistical analysis.

\begin{tabular}{|c|c|c|c|c|c|}
\hline \multirow[t]{2}{*}{ Group } & \multicolumn{3}{|c|}{ Endodontic Pressure Score f(\%) } & \multirow[t]{2}{*}{ X2 Value } & \multirow[t]{2}{*}{ P-Value } \\
\hline & Under & Optimum & Over & & \\
\hline Study Group & & & & 6.251 & 0.405 \\
\hline Group A & $2(10.0)$ & $10(50.0)$ & $8(40.0)$ & & \\
\hline Group B & $3(15.0)$ & $10(50.0)$ & $7(35.0)$ & & \\
\hline Group C & $7(35.0)$ & $9(45.0)$ & $4(20.0)$ & & \\
\hline Group D (Control) & $7(35.0)$ & $8(40.0)$ & $5(25.0)$ & & \\
\hline
\end{tabular}




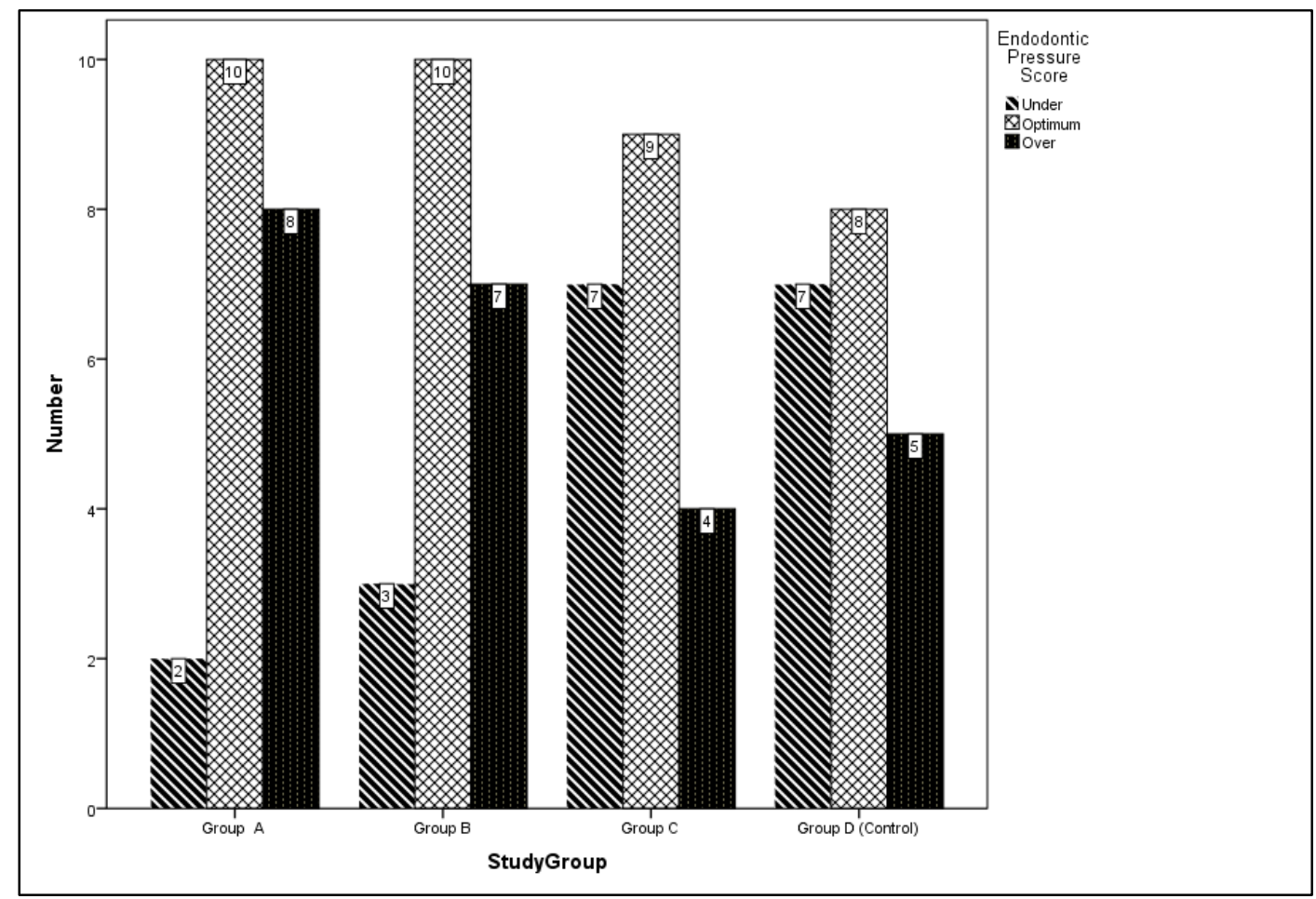

Fig. (1): Number of under, optimum, and over score of length of obturation in ecah group

Table (2): Comparison of score between control and group A, B, C by*Pearson Chi-squared test and **Fishers' exact test were performed for statistical analyses.

\begin{tabular}{|c|c|c|c|c|c|}
\hline \multirow[t]{2}{*}{ Study Groups } & \multicolumn{3}{|c|}{ Score } & \multirow[t]{2}{*}{ X2 Value } & \multirow[t]{2}{*}{ P-Value } \\
\hline & Under & Optimum & Over & & \\
\hline Study Group & & & & 3.572 & $0.218^{\star *}$ \\
\hline Group A & $2(10.0)$ & $10(50.0)$ & $8(40.0)$ & & \\
\hline Group D (Control) & $7(35.0)$ & $8(40.0)$ & $5(25.0)$ & & \\
\hline Study Group & & & & 2.156 & $0.340^{*}$ \\
\hline Group B & $3(15.0)$ & $10(50.0)$ & $7(35.0)$ & & \\
\hline Group D (Control) & $7(35.0)$ & $8(40.0)$ & $5(25.0)$ & & \\
\hline Study Group & & & & 0.263 & $1.000^{\star *}$ \\
\hline Group C & $7(35.0)$ & $9(45.0)$ & $4(20.0)$ & & \\
\hline Group D (Control) & $7(35.0)$ & $8(40.0)$ & $5(25.0)$ & & \\
\hline
\end{tabular}

\section{DISCUSSION}

Pulpectomy refers to the complete removal of irreversibly inflamed or necrotic coronal and radicular pulp tissue followed by filling with a resorbable material in either single or double appointments (Allen, 1979).

An ideal filling technique should assure complete filling of the canal, without overfill or under fill. Overfilled canals lead to mild foreign body reaction and a risk of deflection of succedaneous tooth (Guelmann, 2004). Coll and Sadrian, 1996. found that ZOE-retained material altered the paths of eruption of succedaneous teeth in $20 \%$ of the cases. An under filled canal leads to furcal radiolucency and periapical grauloma and cyst (Ingle and Bakland, 2003).

Coll and Sadrian reported a significant success rate for teeth filled to the apex (89\%) and teeth filled short (87\%) compared to overfilled teeth, which had a $58 \%$ success rate (Coll and Sadrian, 1996).

Several laboratory methods have been used to evaluate the quality of root canal filling, such as the penetration of dye/bacteria/radioisotopes, clearing techniques following tooth sectioning and radiographic assessment (Dandashi etal, 1993). In the present study, the quality of root 
canal obturation was assessed by conventional IOPA radiography.

In the present study, navitip and endodontic pressure syringe performed the best with maximum number of optimal fillings $(50.0 \%)$. (Aylard and Johnson, 1987) have also reported significantly better results with endodontic pressure syringe when compared with the mechanical syringe for obturating the curved root canals. The advantage of endodontic pressure syringe is the screw mechanism which generates pressure that helps in pushing the material into the root canals. However, few drawbacks are also associated with endodontic pressure syringe, namely difficulties in placing the rubber stop correctly and removing the needle, the need to clean and refill the hub of the syringe several times makes this method more complex and time consuming (Memarpour etal, 2013). NaviTip syringe produced the best results in controlling paste extrusion from the apical foramen and having the smallest void size and lowest number of voids (Memarpour etal, 2013).

In the present study, Insulin syringe on the other hand is cost-effective and is easy to use as a carrier for obturating primary teeth (Nagar etal, 2011). It has a small and slender hub that provides an easy access to the canal orifice. insulin syringe group showed (45.0\%) optimum fillings. However, no statistically significant differences were found when compared to remear (control group) $(P=1.000)$ and compared endodontic pressure syringe to remear $(P=0.218)$ also navitip $(P=340)$. The results of the study by Priya Nagar et al showed that the obturation quality of both the reamer technique and insulin syringe technique was found to be very closely related (Nagar etal, 2011).

Endodontic reamear is a simple and effective technique for incremental packing of obturating material into the long and straight canals of primary anterior teeth (Pandranki etal, 2017). However, due to its limited flexibility, it is difficult to obturate a narrow and curved canal till apical portion (Memarpour etal, 2013).

Ravindranath Reddy (Ravindranath Reddy etal, 2015). stated that there is no significant difference among incremental filling (reamer) and endodontic pressure syringe technique used for obturation of primary teeth canals. It was concluded that operator's manual dexterity was very important than the technique of obturation.
In the present study, all the techniques used to obturate the root canals led to voids in the filling material, a finding consistent with earlier reports (Guelmann etal, 2004). However, there were no significant differences $(P>0.05)$ in voids among the four groups studied. Dandashi et al. reported fewer voids with endodontic pressure syringe technique when compared to the reamer, insulin syringe (Dandashi etal, 1993). However, Memarpour et al. found the highest frequency of voids $(87 \%)$ with endodontic pressure syringe and packing groups (Memarpour etal, 2013).

The discrepancies in the results among the studies probably reflects differences in the type of teeth, sample size, needle thickness, and technique used to quantify voids and operator experience (Guelmann etal, 2004). In the present study, the assessment of root canal obturation quality and voids were done by conventional IOPA radiography, which gave a twodimensional view only. This can be the potential limitation of the study. Therefore, it is recommended to conduct in vivo studies with large sample size and long-term follow-up. It is also recommended to use cone-beam computed tomography to accurately assess the quality of root canal obturation.

\section{CONCLUSION}

Navitip and endodontic pressure syringe produced the best results in terms of length of obturation and controlling paste extrusion from the apical foramen. However, insulin syringe and reamer can be used as effective alternative methods.

\section{REFERENCES}

Allen K.R. (1979). Endodontic treatment of primary teeth. Aust Dent J, 24:347-51.

Aylard S.R. and Johnson R. (1987). Assessment of filling techniques for primary teeth. Pediatr Dent, 9(3):195-198.

Berechet, D., Rad, I. A., Berce, C. P., Bumbu, B. A., VICAŞ, R. M., Berechet, M. C., and Cimpean, S. I. (2018). A micro-computed tomography study of morphological aspect of root canal instrumentation with ProTaper Next and One Shape New Generation in mandibular molars. Rom J Morphol Embryol, 59(2), 499-503.

Coll J A. and Sadrian R. (1996). Predicting pulpectomy success and relationship to 
exfoliation and succedaneous dentition. Pediatr Dent, 18:57-63.

Dandashi M.B., Nazif M.M., Zullo T., Elliott M.A., Schneider L.G., and Czonstkowsky M. (1993). An in vitro comparison of three endodontic techniques for primary incisors. Pediatr Dent, 15:254-6.Elnagar M. H., Ghoname, N. A., and Ghoneim, W. M. (2018). Cleaning efficacy of rotary versus manual system for root canal preparation in primary teeth. Tanta Dent J, 15(1), 14-18.

Gesi A. and Bergnholtz G. (2003). Pulpectomy studies on outcome, Endo Topics, 5: 57-70.

Guelmann M., McEachern M., and Turner C. (2004). Pulpectomies in primary incisors using three delivery systems: An in vitro study. J Clin Pediatr Dent, 28:323-6.

Gutmann J.L., Kuttler S., Niemczyk S. (2010). Root Canal Obturation: An Update. Pennwell Publications, 1-11.

Ingle and Bakland (2003). Endodontic diagnostic procedures. Endodontics 5th Edition, 212- 218.

Jha M, Patil SD, Sevekar S, Jogani V, and Shingare P. (2011). Pediatric Obturating Materials and Techniques. Journal of Contemporary Dentistry, 1(2):27- 32.

Jha M., Patil S.D., Sevekar S., Jogani V., Shingare P. (2011). Pediatric Obturating Materials And Techniques. Journal of Contemporary Dentistry, 1(2):27- 3Markovic D., Zivinovic V., and Vucetic M. (2005). Evaluation of three pulpotomy medicaments in primary teeth. Eur $\mathbf{J}$ Paediatr Dent, 6(3): 133-8.
Memarpour M., Shahidi S., and Meshki R. (2013). Comparison of different obturation techniques for primary molars by digital radiography. Pediatr Dent, 35:236-40.

Moutaz B., Dandashi, Mamoun M., Nazif, Thomas Zullo, Margaret A. Elliott, Lawrence G. Schneider and Mario Czonstkowsky (1993). An in vitro comparison of the three endodontic techniques for primary incisors. Pediatr Dent, 15(4):254-256.

Nagar P., Araali V., and Ninawe N. (2011) An alternative obturation technique using insulin syringe delivery systemto traditional reamer: an in-vivo study. J Dent Oral Biosci, 2(2):7-9.

Pandranki J., Chitturi R.R., Vanga N.R., and Chandrabhatla S.K. (2017) A comparative assessment of different techniques for obturation with endoflas in primary molars: an in vivo study. Indian J Dent Res, 28(1):44-48.

Ravindranath Reddy P. V., Shivayogi M. Hugar, Anand Shigli, Suganya M, Shweta S Hugar and Pratibha Kukreja (2015). Comparative evaluation of efficiency of three obturation techniques for primary incisors-An in vivo study. International Journal of Oral Health and Medical Research. July-August; 2(2):15-18.

Reddy R., Hugar S.M., and Shigli A., Suganya M., Hugar S.S., and Kukreja P. (2015). Comparative Evaluation of Efficiency of Three Obturation Techniques for Primary IncisorsAn In Vivo Study. IJOHMR J, 2: 2 